\title{
ON VECTOR LYAPUNOV FUNCTIONS ${ }^{1}$
}

\author{
S. G. $\mathrm{DEO}^{2}$
}

\begin{abstract}
ABsTRACr. It has been proved that the use of a vector Lyapunov function is more advantageous in certain situations rather than a scalar function. Moreover, each function needs to satisfy less rigid requirements. In this paper a new situation has been considered where vector Lyapunov functions play a further useful role. For this purpose, a new type of stability, namely, strict partial stability has been defined. The principal tool employed is the second method of Lyapunov and a comparision theorem of a more general type.
\end{abstract}

1. Introduction. In an interesting paper Bellman [1] has raised a question whether it might be more convenient, in certain situations, to use a vector Lyapunov function rather than a scalar function. The answer is positive and it has been pointed out that the application of several Lyapunov functions is indeed useful [4], [5]. The object of the present paper is to extend this idea further.

Lyapunov's principal theorems give sufficient conditions for the stability, asymptotic stability and instability of systems. During the last few years these stability concepts have been refined and further generalized in several directions. In particular, the concept of partial stability has been studied by Corduneanu [3]. This type of stability is useful from the practical point of view since in many situations one is interested in the behaviour of some variables only. This idea suggests that it is possible to consider the behaviour of the remaining variables and thus this approach leads to the concept of strict partial (s.p.) stability.

While proving the main results in [3], a differential inequality has been employed to study the partial stability properties. This technique involves estimation of a function, satisfying differential inequality, by the maximal solution of a related differential system. Such differential inequalities have been used in considerable details to solve a number of problems in the analysis of systems and the most comprehensive text for such a literature is [4].

Received by the editors August 3, 1970 and, in revised form, November 2, 1970.

AMS 1970 subject classifications. Primary 34D20; Secondary 34A40.

Key words and phrases. Vector Lyapunov function, minimax solution, partial stability.

${ }^{1}$ This work was supported by N.R.C. Canada operating grants A-3072 and A3074.

${ }^{2}$ On leave from Marathwada University, Aurangabad, India. 
Following the above approach, it is believed that the other type of extremal solution, namely the minimax solution, has not yet been exploited to study the properties of differential systems. The existence of this type of critical solution has been proved by Burton and Whyburn [2] under certain monotonic conditions. We employ below the second method of Lyapunov and the minimax solution of the related system to study the strict partial stability properties of differential systems.

2. Notation and definitions. Let $J$ denote the interval $0 \leqq t<\infty$ and $R^{n}$ - the $n$-dimensional Euclidean space. Let $H_{\tau}=\left\{z \in R^{n},\|z\|<\tau\right.$, $\tau>0\}$ where $\|\cdot\|$ denotes a suitable norm in $R^{n}$. Consider a differential system

$$
\dot{z}=Z(t, z)
$$

or a more explicit form

$$
\dot{x}=X(t, z), \quad \dot{y}=Y(t, z),
$$

where $x, X \in R^{p}, y, Y \in R^{q}, p+q=n$ and $Z$ is a real $n$-vector function defined and continuous on $J \times H_{\tau}$. Suppose that the function $Z$ is sufficiently smooth in order that, for every $\left(t_{0}, z_{0}\right) \in J \times H_{\tau}$, there exists a solution $z\left(t ; t_{0}, z_{0}\right)=\left(x\left(t ; t_{0}, z_{0}\right), y\left(t ; t_{0}, z_{0}\right)\right)$ depending continuously on $t_{0}, z_{0}$ and equal to $z_{0}$ at $t_{0}$. We do not assume the uniqueness of solutions of the system (2.1). Let $Z(t, 0)=0$, for every $t \in J$, so that (2.1) admits the trivial solution.

We say that a real valued function $a(r)$ belongs to class $K$ if it is defined, continuous and strictly increasing on $0 \leqq r<\infty$ and if it vanishes at $r=0: a(0)=0$. Our interest lies in the following stability properties. The trivial solution is said to be:

$\left(\mathrm{P}_{1}\right)$ s.p. equistable if given $0<\epsilon<\tau$ and $t_{0} \in J$, there exists a function $\delta=\delta\left(t_{0}, \epsilon\right)$ which is continuous in $t_{0}$ for each $\epsilon$ and a $t_{1}>t_{0}$ such that $\left\|x\left(t ; t_{0}, z_{0}\right)\right\|<\epsilon$ for $t \geqq t_{0}$ and $\left\|y\left(t_{1} ; t_{0}, z_{0}\right)\right\| \geqq \epsilon$ whenever $\left\|z_{0}\right\| \leqq \delta$;

$\left(\mathrm{P}_{2}\right)$ s.p. uniformly stable if the $\delta$ in $\left(\mathrm{P}_{1}\right)$ is independent of $t_{0}$;

$\left(\mathrm{P}_{3}\right)$ s.p. quasi-equi-asymptotically stable if, for each $0<\epsilon<\tau, t_{0} \in J$, there exist positive numbers $\delta_{0}=\delta_{0}\left(t_{0}\right), T=T\left(t_{0}, \epsilon\right)$ and $t_{1}>t_{0}$ such that $\left\|x\left(t ; t_{0}, z_{0}\right)\right\|<\epsilon$ for $t \geqq t_{0}+T$ and $\left\|y\left(t_{1} ; t_{0}, z_{0}\right)\right\| \geqq \epsilon$ whenever $\left\|z_{0}\right\| \leqq \delta_{0}$;

$\left(\mathrm{P}_{4}\right)$ s.p. quasi-uniformly asymptotically stable if the numbers $\delta_{0}$ and $T$ in $\left(\mathrm{P}_{3}\right)$ are independent of $t_{0}$;

$\left(\mathrm{P}_{5}\right)$ s.p. equi-asymptotically stable if $\left(\mathrm{P}_{1}\right)$ and $\left(\mathrm{P}_{3}\right)$ hold simultaneously;

$\left(\mathrm{P}_{6}\right)$ s.p. uniformly asymptotically stable if $\left(\mathrm{P}_{2}\right)$ and $\left(\mathrm{P}_{4}\right)$ hold simultaneously. 
3. Related system. Let $\lambda(t, u)$ be a 2-vector function defined and continuous on the product space $J \times R^{2}$. Let $\lambda_{1}\left(t, u_{1}, u_{2}\right)$ be monotone decreasing in $u_{2}$ for each $\left(t, u_{1}\right)$ and $\lambda_{2}\left(t, u_{1}, u_{2}\right)$ be monotone decreasing in $u_{1}$ for each $\left(t, u_{2}\right)$. Consider the differential system

$$
\dot{u}=\lambda(t, u), \quad u\left(t_{0} ; t_{0}, u_{0}\right)=u_{0} ; \quad\left(t \geqq t_{0}\right) .
$$

We note that the system (3.1), in general, may not possess the maximal and minimal solutions since the existence of these extremal solutions need that $\lambda(t, u)$ should be quasi-monotonic in $u$ [2]. However, it has another critical solution known as minimax (1 max-1 min) solution. It has been proved in [2] that under the given monotonicity conditions the minimax solution for the system (3.1) exists.

Further, let $S=J \times D$ where $D$ is an open set in $H_{\tau}$ containing the origin and let $v=\left[v_{1}, v_{2}\right]$ be such that

$$
v_{1} \in C\left[J \times H_{\tau}, R^{+}\right] \text {and } v_{2} \in C\left[\bar{S}, R^{+}\right] ;
$$

where $\bar{S}$ denotes the closure of $S$;

(3.3) $v_{1}(t, z) \rightarrow 0$ as $\|z\| \rightarrow 0$ for each $t \in J$, it is locally Lipschitzian in $z$;

(3.4) $v_{2}(t, z)=0$ for $z \in \bar{S}-S, v_{2}$ is bounded on $\bar{S}$ and locally Lipschitzian in $z$.

Define, for $(t, z) \in \bar{S}$,

$$
v^{*}(t, z)=\limsup _{h \rightarrow 0^{+}} \frac{1}{h}[v(t+h, z+h Z(t, z))-v(t, z)] .
$$

The principal tool employed to prove the main results is the following lemma.

Lemma. Let the vector function $\lambda(t, u)$ in (3.1) be such that $\lambda_{2}(t, u) \geqq 0$. Let the function $v(t, z)$ satisfy conditions (3.2), (3.3) and (3.4). Suppose that $u\left(t ; t_{0}, u_{0}\right)$ is the minimax solution of (3.1) existing for $t \geqq t_{0}$ and the function $v^{*}(t, z)$ of $(3.5)$ satisfies the inequalities

$$
(-1)^{i+1} v_{i}^{*}(t, z) \leqq(-1)^{i+1} \lambda_{i}(t, v(t, z)) \quad(i=1,2)
$$

for $(t, z) \in S$. Let $z\left(t ; t_{0}, z_{0}\right)$ be any solution of $(2.1)$ such that

$$
(-1)^{i+1} v_{i}\left(t_{0}, z_{0}\right) \leqq(-1)^{i+1} u_{i 0}, \quad\left(t_{0}, z_{0}\right) \in S .
$$

Then

$$
(-1)^{i+1} v_{i}\left(t, z\left(t ; t_{0}, z_{0}\right)\right) \leqq(-1)^{i+1} u_{i}\left(t ; t_{0}, u_{0}\right) \quad(i=1,2)
$$

for $(t, z) \in S$. 
REMARK. The proof of this lemma can be formulated by following the argument of Theorem 1 of [2]. The details are omitted. However, it is important to note that the solution $z\left(t ; t_{0}, z_{0}\right)$ belongs to $S$ under the given conditions. For, choose $\left(t_{0}, z_{0}\right) \in S, z_{0} \neq 0$; then, due to (3.6),

$$
v_{2}^{*}(t, z) \geqq \lambda_{2}\left(t, v_{1}(t, z), v_{2}(t, z)\right) \geqq 0 .
$$

Further, since $v_{2}$ is Lipschitzian by hypothesis, we have

$$
v_{2}\left(t, z\left(t ; t_{0}, z_{0}\right)\right) \geqq v_{2}\left(t_{0}, z_{0}\right)>0 .
$$

Further, since $v_{2}(t, z)=0$ for all $(t, z) \in \bar{S}-S$, it follows from the inequality (3.9) that $\left(t, z\left(t ; t_{0}, z_{0}\right)\right) \in S$ for $t \geqq t_{0}$.

Now, let $u\left(t ; t_{0}, u_{0}\right)$ be any solution of (3.1). Corresponding to properties $\left(\mathrm{P}_{1}\right)$ to $\left(\mathrm{P}_{6}\right)$ we can formulate properties $\left(\mathrm{P}_{1}^{*}\right)$ to $\left(\mathrm{P}_{6}^{*}\right)$ for the system (3.1). For example, corresponding to $\left(\mathrm{P}_{1}\right)$, we have:

$\left(\mathrm{P}_{1}^{*}\right)$ given $0<\epsilon<\tau$ and $t_{0} \in J$, there exists a function $d=d\left(t_{0}, \epsilon\right)$, which is continuous in $t_{0}$ for each $\epsilon$ and a $t_{1}>t_{0}$ such that $u_{1}\left(t ; t_{0}, u_{0}\right)$ $<\epsilon$ for $t \geqq t_{0}$ and for arbitrary small $u_{20}>0, u_{2}\left(t ; t_{0}, u_{0}\right)$ is either unbounded or indeterminate whenever $u_{10} \leqq d$ and $u_{20}>0$.

4. Main results. We now establish the following theorems which give sufficient conditions for properties $\left(\mathrm{P}_{1}\right)$ to $\left(\mathrm{P}_{6}\right)$ to hold in terms of vector Lyapunov functions.

THEOREM 1. Let the assumptions of the lemma hold and let the vector function $v$ described above be such that, for $(t, z) \in J \times H_{\tau}$,

$$
b(\|x\|) \leqq v_{1}(t, z), \quad b \in K .
$$

Then the properties $\left(\mathrm{P}_{1}^{*}\right),\left(\mathrm{P}_{3}^{*}\right)$ and $\left(\mathrm{P}_{5}^{*}\right)$ imply the corresponding properties $\left(\mathrm{P}_{1}\right),\left(\mathrm{P}_{3}\right)$, and $\left(\mathrm{P}_{5}\right)$ respectively.

Proof. Let $0<\epsilon<\tau$. Since $\left(\mathrm{P}_{1}^{*}\right)$ holds, given $b(\epsilon)>0, t_{0} \in J$, there exists a positive function $d=d\left(t_{0}, \epsilon\right)$, which is continuous in $t_{0}$ for each $\epsilon$, such that

$$
u_{1}\left(t ; t_{0}, u_{0}\right)<b(\epsilon), \quad t \geqq t_{0}
$$

whenever

$$
u_{10} \leqq d \text { and } u_{20}>0 .
$$

Since the assumptions of the lemma hold, we have, for $\left(t, z\left(t ; t_{0}, z_{0}\right)\right)$ $\in S$,

$$
v_{1}\left(t, z\left(t ; t_{0}, z_{0}\right)\right) \leqq u_{1}\left(t ; t_{0}, u_{0}\right)
$$

whenever 


$$
v_{1}\left(t_{0}, z_{0}\right) \leqq u_{10} ; \quad\left(t_{0}, z_{0}\right) \in S .
$$

We choose $v\left(t_{0}, z_{0}\right)=u_{0}$; then the fact that $v_{1}(t, z)$ is continuous and $v_{1}(t, z) \rightarrow 0$ as $\|z\| \rightarrow 0$ for each $t$ yields, in view of (4.3) and (4.5), that there exists a $\delta=\delta\left(t_{0}, \epsilon\right)$, which is continuous in $t_{0}$ for each $\epsilon$, such that the inequalities

$$
\left\|z_{0}\right\| \leqq \delta, \quad v_{1}\left(t_{0}, z_{0}\right) \leqq d,
$$

hold simultaneously. We now show that with this $\delta$ the property $\left(\mathrm{P}_{1}\right)$ holds. For, otherwise, there exists a $\tilde{t}>t_{0}$ for which

$$
\left\|x\left(\tilde{t} ; t_{0}, z_{0}\right)\right\|=\epsilon \text { and }\left\|x\left(t ; t_{0}, z_{0}\right)\right\| \leqq \epsilon, \quad t_{0} \leqq t \leqq \tau,
$$

whenever $\left\|z_{0}\right\| \leqq \delta$. But then we find

$$
b(\epsilon) \leqq v_{1}\left(\tilde{t}, z\left(\tilde{t} ; t_{0}, z_{0}\right)\right) \leqq u_{1}\left(\tilde{t} ; t_{0}, u_{0}\right)<b(\epsilon)
$$

by virtue of (4.1), (4.2) and (4.4), which is impossible. Also, we have, for $(t, z) \in S$,

$$
v_{2}\left(t, z\left(t ; t_{0}, z_{0}\right)\right) \geqq u_{2}\left(t ; t_{0}, u_{0}\right),
$$

by (3.8). Further, since $v_{2}(t, z)$ is bounded by assumption, according to property $\left(\mathrm{P}_{1}^{*}\right)$, the inequality (4.6) cannot hold if we assume that the trivial solution of (2.1) is stable. Thus, the trivial solution is stable with respect to $x$-components but it is unstable with respect to $y$-components. This proves that the property $\left(\mathrm{P}_{1}\right)$ holds. To prove that $\left(\mathrm{P}_{3}^{*}\right)$ implies $\left(\mathrm{P}_{3}\right)$, let $b(\epsilon)>0, t_{0} \in J$, be given. Then there exist positive numbers $d_{0}=d_{0}\left(t_{0}\right)$ and $T=T\left(t_{0}, \epsilon\right)$ such that

$$
u_{1}\left(t ; t_{0}, u_{0}\right)<b(\epsilon), \quad t \geqq t_{0}+T,
$$

whenever $u_{10} \leqq d$ and $u_{20}>0$.

As in the previous case, we choose a number $\delta_{0}$. Now, suppose that there is a divergent sequence $\left\{t_{k}\right\}, t_{k} \geqq t_{0}+T$, such that $\left\|x\left(t_{k} ; t_{0}, z_{0}\right)\right\|$ $=\epsilon$ whenever $\left\|z_{0}\right\| \leqq \delta_{0}$. Then, in view of (4.1), (4.4) and (4.7), we have

$$
b(\epsilon) \leqq v_{1}\left(t_{k}, z\left(t_{k} ; t_{0}, z_{0}\right)\right) \leqq u_{1}\left(t_{k} ; t_{0}, u_{0}\right)<b(\epsilon)
$$

which is impossible. This proves that $\left\|x\left(t ; t_{0}, z_{0}\right)\right\|<\epsilon$ for $t \geqq t_{0}+T$. As in the previous case, we can show that the trivial solution is unstable with respect to $y$-components. This proves that $\left(\mathrm{P}_{3}^{*}\right)$ holds.

Now, it is easy to conclude that $\left(\mathrm{P}_{5}^{*}\right)$ implies property $\left(\mathrm{P}_{5}\right)$.

THEOREM 2. Let the assumptions of the lemma hold and let the vector function $v$ described above be such that, for $(t, z) \in J \times H_{r}$,

$$
b(\|x\|) \leqq v_{1}(t, z) \leqq a(\|z\|), \quad a, b \in K .
$$


Then the properties $\left(\mathrm{P}_{2}^{*}\right),\left(\mathrm{P}_{4}^{*}\right)$ and $\left(\mathrm{P}_{6}^{*}\right)$ imply the corresponding properties $\left(\mathrm{P}_{2}\right),\left(\mathrm{P}_{4}\right)$ and $\left(\mathrm{P}_{6}\right)$ respectively.

Proof. The proof is similar to that of Theorem 1 except that the function $a \in K$ assumed in (4.8) helps to determine $\delta$ in $\left(\mathrm{P}_{1}\right), \delta_{0}$ and $T$ in $\left(\mathrm{P}_{2}\right)$ respectively, independent of $t_{0}$. The details are omitted.

Acknowledgment. The author acknowledges with thanks the fruitful suggestions made by the referee.

\section{REFERENCES}

1. R. E. Bellman, Vector Lyapunov functions, J. SIAM Control Ser. A 1 (1962), 32-34. MR $26 \# 414$.

2. L. P. Burton and W. M. Whyburn, Minimax solutions of ordinary differential systems, Proc. Amer. Math. Soc. 3 (1952), 794-803. MR 14 \#470.

3. C. Corduneanu, Sur la stabilité partielle, Rev. Roumaine Math. Pures Appl. 9 (1964), 229-236. MR 31 \#4956.

4. V. Lakshmikantham and S. Leela, Differential and integral inequalities. Vols. I, II, Academic Press, New York, 1969.

5. V. M. Matrosov, The comparision principle with a vector-valued Lyapunov function. I, Differencial'nye Uravnenija 4 (1968), 1374-1386. (Russian) MR 38 \#2361.

University of Alberta, Edmonton, Alberta, Canada 